\title{
Digital Simulation of Superconductive Memory System Based on Hardware Description Language Modeling
}

\author{
S. Narendran (iD) and J. Selvakumar \\ Department of Electronics \& Communication Engineering, SRM Institute of Science and Technology, Chennai, India \\ Correspondence should be addressed to S. Narendran; naren.sridhar@hotmail.com
}

Received 11 February 2018; Accepted 24 April 2018; Published 27 May 2018

Academic Editor: Sergei Sergeenkov

Copyright (c) 2018 S. Narendran and J. Selvakumar. This is an open access article distributed under the Creative Commons Attribution License, which permits unrestricted use, distribution, and reproduction in any medium, provided the original work is properly cited.

\begin{abstract}
We have modeled a memory system using Josephson Junction to attain low power consumption using low input voltage compared to conventional Complementary Metal Oxide Semiconductor-Static Random Access Memory (CMOS-SRAM). We attained the low power by connecting a shared/common bit line and using a 1-bit memory cell. Through our design we may attain 2.5-3.5 microwatts of power using lower input voltage of 0.6 millivolts. Comparative study has been made to find which memory system will attain low power consumption. Conventional SRAM techniques consume power in the range of milliwatts with the supply input in the range of 0-10 volts. Using HDL language, we made a memory logic design of RAM cells using Josephson Junction in FreeHDL software which is dedicated only for Josephson Junction based design. With use of XILINX, we have calculated the power consumption and equivalent Register Transfer Level (RTL) schematic is drawn.
\end{abstract}

\section{Introduction}

For low power consumption and high performance computing, Single flux Quantum (SFQ) is widely used, which is an alternate principle for Complementary Metal Oxide Semiconductor (CMOS) technology. Many SFQ techniques are devolved to attain high speed and low power consumption. Various approaches have been developed on Josephson, CMOS hybrid memory cell, which is high density CMOS memory array and low power Single flux Quantum (SFQ) logic [1-4]. In addition, the hybrid model must work at cryogenic temperature. The $64 \mathrm{~kb}$ hybrid static CMOS RAM has been discussed in [5]; the estimated power consumption of this hybrid logic is $20 \mathrm{~mW}$ for read and $53.7 \mathrm{~mW}$ for write operation. In [6], further reduction on power consumption is achieved by introducing a binary tree decoder and with help of data driver, they attain $54 \%$ and $8 \%$ of write and read operation. We attain large scale high speed memory using cryogenic experience to match the ultra-speed processor in the same cryogenic temperature. Many developments and demonstrations of hybrid memory logic have been evolved using SFQ and RSFQ (Rapid Single Flux Quantum) logic.

In this paper, we developed a RAM using Reciprocal Quantum Logic at cryogenic temperature. The proposed memory system is of static nature which is composed of decoder and sense amplification circuit. In Section 2, we have discussed convention SRAM using 6T and 8T transistor. We have designed a RAM using RQL which is discussed in Section 3. Comparative study and results are discussed in Section 4 and we have concluded our remarks in Section 5.

\section{Conventional CMOS Memory Cell Design}

The most commonly used control signals in CMOS RAM cell are Write Enable (WE), Output Enable (OE), and Chip Select (CS). Conventional 6T CMOS memory cell consists of four NMOS and two PMOS counts with separate bit line and word line. The average power consumption of 6T SRAM cell is $46 \mathrm{~mW}$ and area required is $5 \mu \mathrm{m}^{2}$. For $8 \mathrm{~T}$ transistor, the dynamic power consumption will be $0.035 \mu \mathrm{W}$. For both 


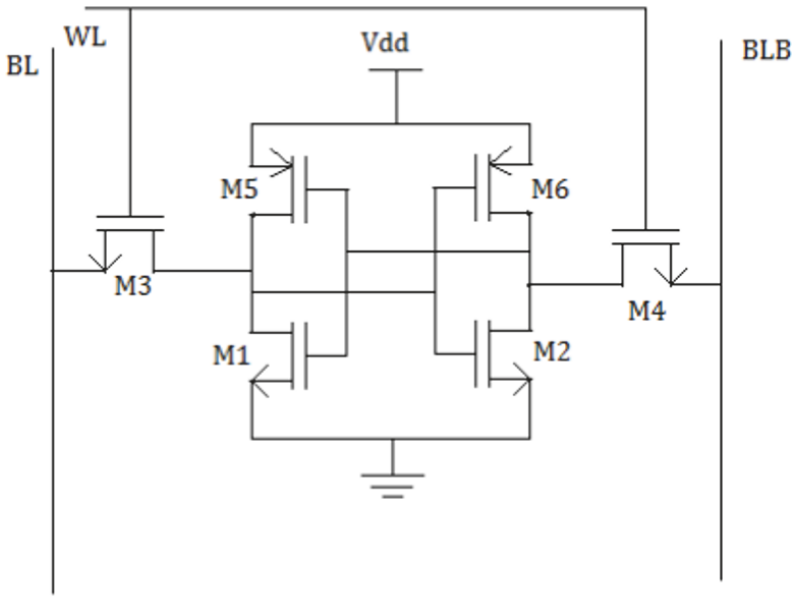

FIgURE 1: Conventional 6T CMOS-SRAM cell [7].

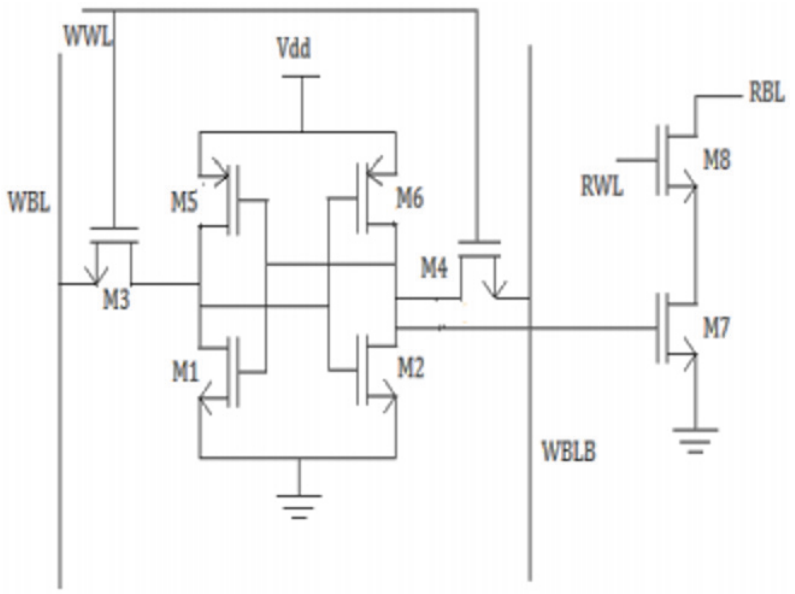

FIGURE 2: Conventional 8T SRAM CMOS cell.

SRAM memory cell, the voltage at both bit lines is precharged before the read operation starts. Consider logic 0 at transistor M3 and logic 1 at M4; the particular memory can be selected using word line and bit line correspondingly according to discharge of current flows from the supply voltage. For write operation, the bit line (BL) will complement its signal from logic 0 to logic 1 and vice versa. The cross coupled inverter structure will generate high gain to interchange the voltages passing through the transistor.

By use of NMOS and PMOS logic, corresponding 6T and 8T SRAM cells are formed in Figures 1 and 2. For 6T SRAM cell, there are four NMOS and two PMOS are used. And, for 8T SRAM CMOS cell it requires six NMOS and two PMOS logic structures. Furthermore, different SRAM architectures have been developed using 7T and 9T CMOS cells.

\section{Superconductive Memory Cell}

In SRAM cell design, they are incorporable in terms of power dissipation due to high capacitance buses. Due to excessive access of capacitance buses, low-voltage low power cell designs are developed in CMOS families. With the help of shared bit line cell configuration of SRAM cell libraries, we can achieve ultra-low power dissipation. MUX based charged sense amplifiers are used for read operation. For write operation, a bit line precharged technique is used to attain high speed write operation [8].

With the help of low-voltage low power (LVLP) technique, the normalized form of RAM cell design is made by replacing MOS technology with Josephson Junction (JJ) technology. Below, Figure 3 shows the structure of RAM architecture by replacing CMOS cell libraries with Reciprocal Quantum Logic structure.

Reciprocal Quantum Logic (RQL) is one of the high speed low power consumption superconductive logics. RQL circuits are designed using alternating current phenomena rather all CMOS or any digital circuits designed using direct current as input. In [9-11], RQL circuits are designed using the basic gates of RQL as ANDOR, ANOTB, Latch circuit, and XOR logic gates. In our design we also used the basic gates to form 
TABLE 1: Comparison of power consumption.

\begin{tabular}{lccccc}
\hline Technology & RQL based RAM & $6 \mathrm{~T}$ & $8 \mathrm{~T}$ & Hybrid JJ- CMOS & Starc JJ - CMOS Hybrid \\
\hline Power for Write $(\mathrm{W})$ & $3.45 \mu \mathrm{W}$ & $46 \mu \mathrm{W}$ & $37.1 \mu \mathrm{W}$ & $27.62 \mathrm{~mW}$ & $11.43 \mathrm{~mW}$ \\
\hline Power for Read $(\mathrm{W})$ & $2.6 \mu \mathrm{W}$ & $44.26 \mu \mathrm{W}$ & $39.9 \mu \mathrm{W}$ & $21.25 \mathrm{~mW}$ & $9.18 \mathrm{~mW}$ \\
\hline
\end{tabular}

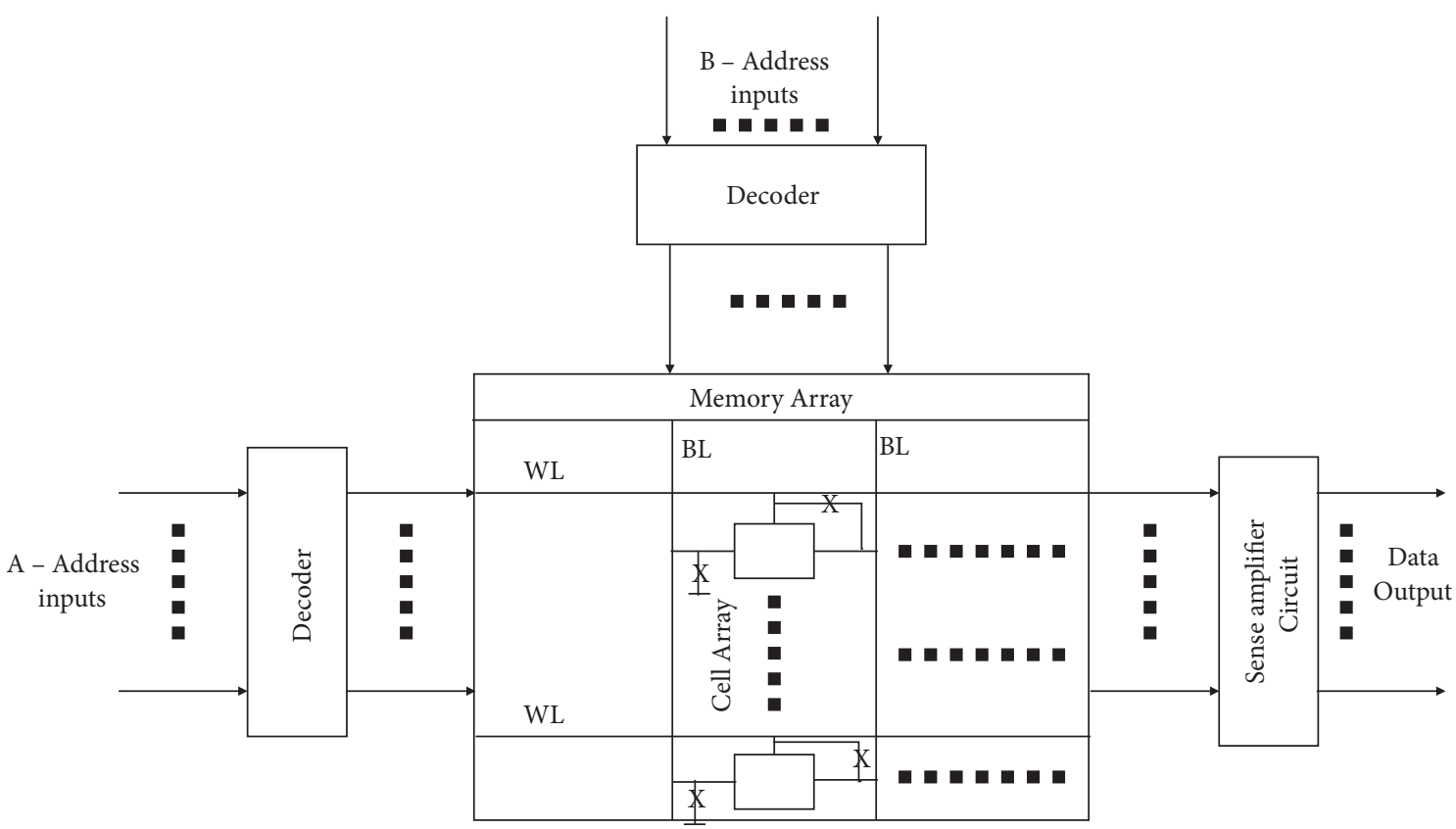

FIgURE 3: Structure of a memory system.

RAM logic to consume less power comparative to the older techniques. Other superconducting logics include RSFQ, AQFP, and eSFQ, which are successor of convention CMOS logic design. Those logics have their own pros and cons. By using RSFQ [12-14], many articles have been published for the design of RAM and processors which can work $25 \%$ to $35 \%$ more in performance comparative to the CMOS RAM cells.

\section{Simulation Results of RQL Based RAM}

With the help of Very High Speed Integrated Circuit (VHSIC) Hardware Description Language (VHDL), the coding of RAM cell is designed and corresponding results are found out. By using XILINX software and FreeHDL software, we have generated the RTL schematic view of our RAM design. Figure 4 shows the Register Transfer Level (RTL) schematic view of RAM cell using RQL.

We have compared our result with previously developed RAM cell like 6T, 8T, Hybrid Josephson Junction- (JJ-) CMOS for $64 \mathrm{~Kb}$, and hybrid binary decoder of JJ-CMOS. We have presented it in Table 1. For the results obtained in Table 1, we made a chart comparison and it is shown in Figure 5.

\section{Conclusion}

We have designed a RAM cell using RQL superconducting logic. We achieved the power consumption of $2.6 \mu \mathrm{W}$ for read operation and $3.45 \mu \mathrm{W}$ for write operation, which is very much less compared to other technologies. We designed the RAM using HDL language and analysed the power using XILINX software. Since we have used the Reciprocal Quantum Logic (RQL), we can further implement this technique in real time application for high speed processor to support high speed applications. Our future work will be designing a processor based on RQL and implementing this RAM technique to that processor.

\section{Data Availability}

The data used to support the findings of this study are available from the corresponding author upon request.

\section{Conflicts of Interest}

The authors declare that there are no conflicts of interest regarding the publication of this paper 


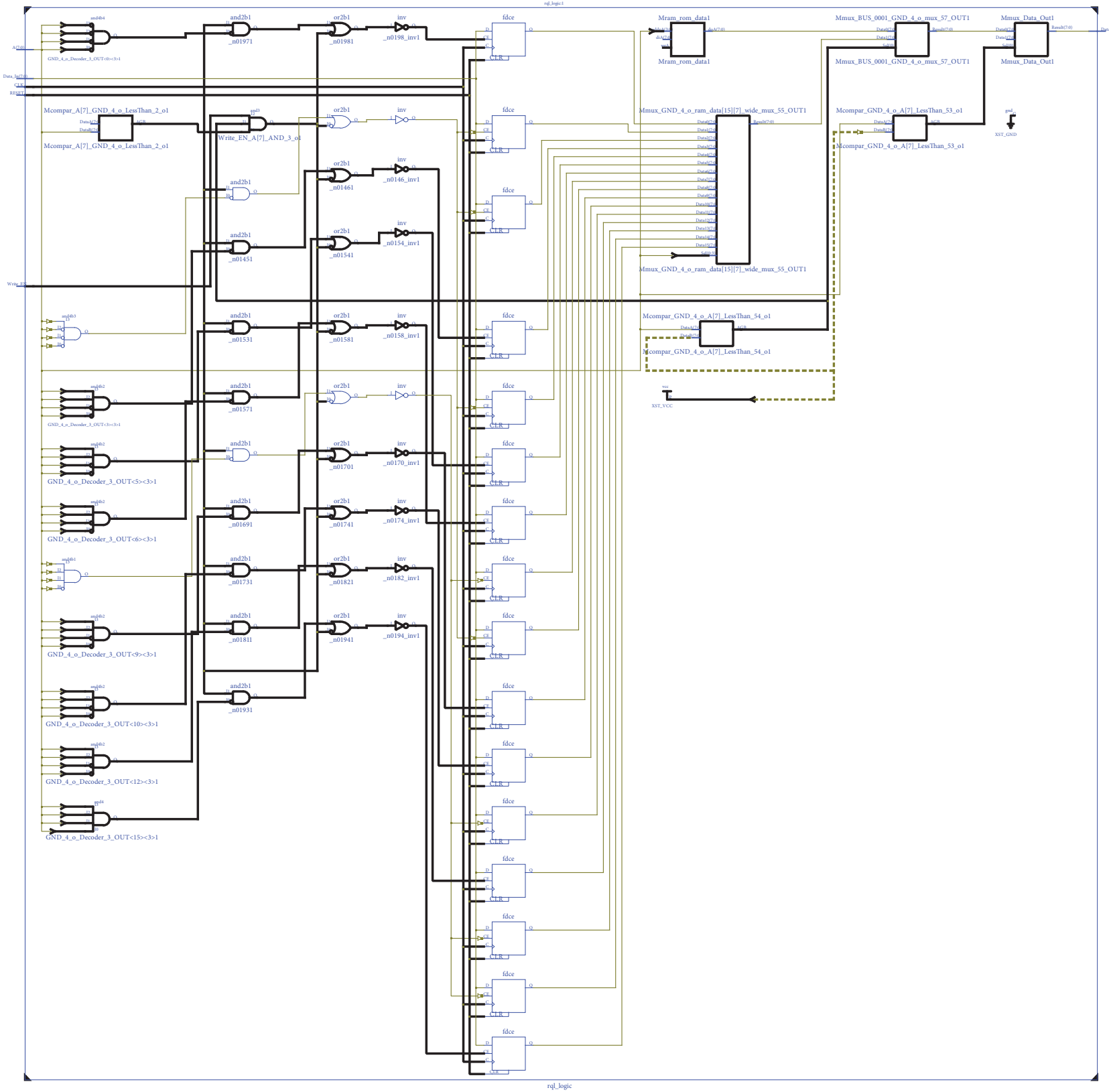

FIGURE 4: RTL schematic view of RAM using Reciprocal Quantum Logic.

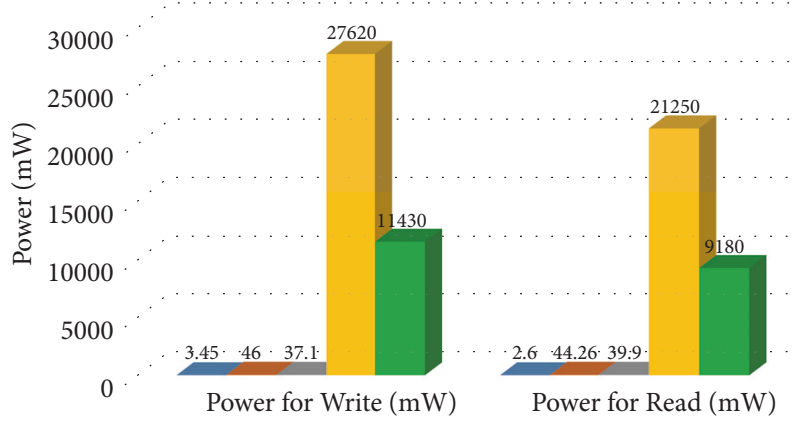

$$
\begin{array}{ll} 
& \text { RQL based RAM } \\
=6 \mathrm{~T} & \text { Hybrid JJ - CMOS } \\
-8 \mathrm{~T} &
\end{array}
$$

FIGURE 5: Power comparison of various technologies. 


\section{References}

[1] U. Ghoshal, H. Kroger, and T. Van Duzer, "Superconductorsemiconductor memories," IEEE Transactions on Applied Superconductivity, vol. 3, no. 1, pp. 2315-2318, 1993.

[2] Q. Liu, T. Van Duzer, X. Meng et al., "Simulation and measurements on a 64-kbit hybrid josephson-CMOS memory," IEEE Transactions on Applied Superconductivity, vol. 15, no. 2, pp. 415-418, 2005.

[3] N. Yoshikawa, T. Tomida, M. Tokuda et al., "Characterization of $4 \mathrm{~K} \mathrm{CMOS}$ devices and circuits for hybrid Josephson-CMOS systems," IEEE Transactions on Applied Superconductivity, vol. 15, no. 2, pp. 267-271, 2005.

[4] N. Yoshikawa and etal., "Access time measurement of Josephson-CMOS hybrid memory using single-flux-quantum circuits," Superconductor Science and Technology, vol. 19, no. 5, pp. S1-S4, May 2006.

[5] K. Kuwabara, H. Jin, Y. Yamanashi, and N. Yoshikawa, "Design and implementation of 64-kb CMOS static RAMs for Josephson-CMOS hybrid memories," IEEE Transactions on Applied Superconductivity, vol. 23, no. 3, Article ID 1700704, 2013.

[6] G. Konno, Y. Yamanashi, and N. Yoshikawa, "Fully functional operation of low-power 64-kb Josephson-CMOS Hybrid Memories," IEEE Transactions on Applied Superconductivity, vol. 27, no. 4, 2017.

[7] M. Gopal, D. Siva Sankar Prasad, and B. Raj, "8T SRAM cell design for dynamic and leakage power reduction," International Journal of Computer Applications, vol. 71, no. 9, pp. 43-48, 2013.

[8] Y. Kiat-Seng and R. Kaushik, Low-Voltage, Low-Power VLSI Subsystems, MCGraw-Hill Professional Engineering, New York, NY, USA, 2005.

[9] O. T. Oberg, Superconducting logic circuits operating with reciprocal magnetic flux quanta [Ph.D. thesis], University of Maryland, 2011.

[10] Q. P. Herr, M. Stoutimore, E. Min, and A. Herr, "Design of a Reciprocal Quantum Logic multiport register file," in Proceedings of the Applied Superconductor Conference, Charlotte, NC, USA, 2014.

[11] P. D. Dresselhaus and etal., "Fabrication of large area RQL circuits with a-Si barrier Josephson junctions," in Proceedings of the Applied Superconductor Conference, Charlotte, NC, USA, 2014.

[12] K. K. Likharev and V. K. Semenov, "RSFQ logic/memory family: a new Josephson-Junction technology for sub-terahertz-clockfrequency digital systems," IEEE Transactions on Applied Superconductivity, vol. 1, no. 1, pp. 3-28, 1991.

[13] M. Tanaka, R. Sato, Y. Hatanaka, and A. Fujimaki, "Highdensity shift-register-based rapid single-flux-quantum memory system for bit-serial microprocessors," IEEE Transactions on Applied Superconductivity, vol. 26, no. 5, 2016.

[14] M. Tanaka, K. Takata, T. Kawaguchi et al., "Development of bit-serial RSFQ microprocessors integrated with shift-registerbased random access memories," in Proceedings of the 2015 15th International Superconductive Electronics Conference (ISEC), Nagoya, Japan, July 2015. 

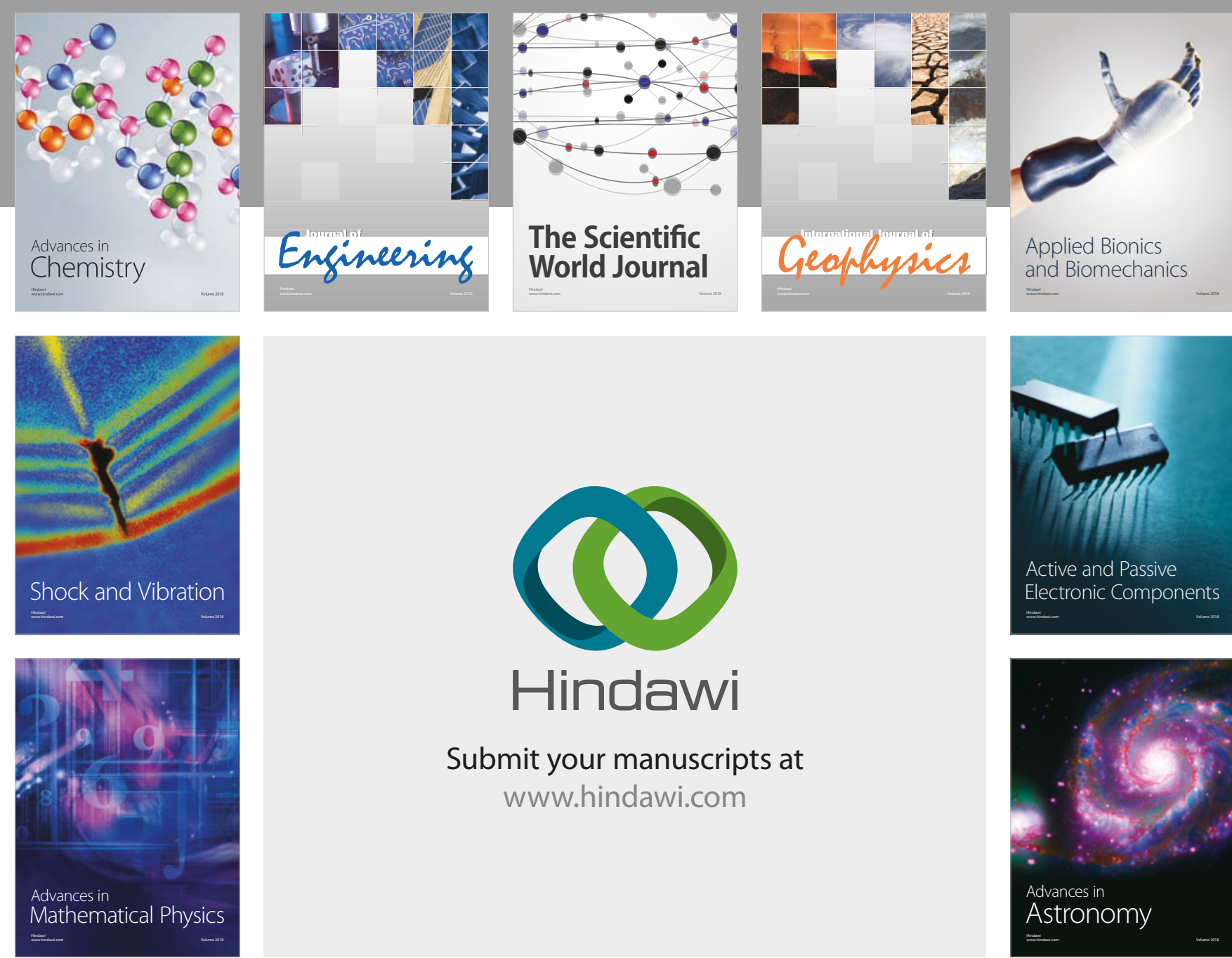

Submit your manuscripts at

www.hindawi.com

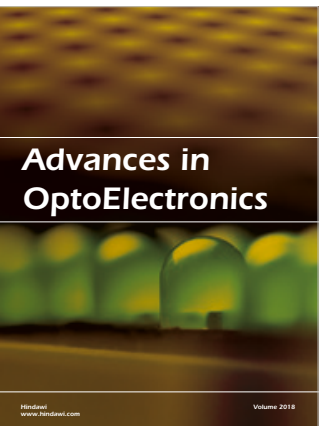

\section{Rotcting Machinery}
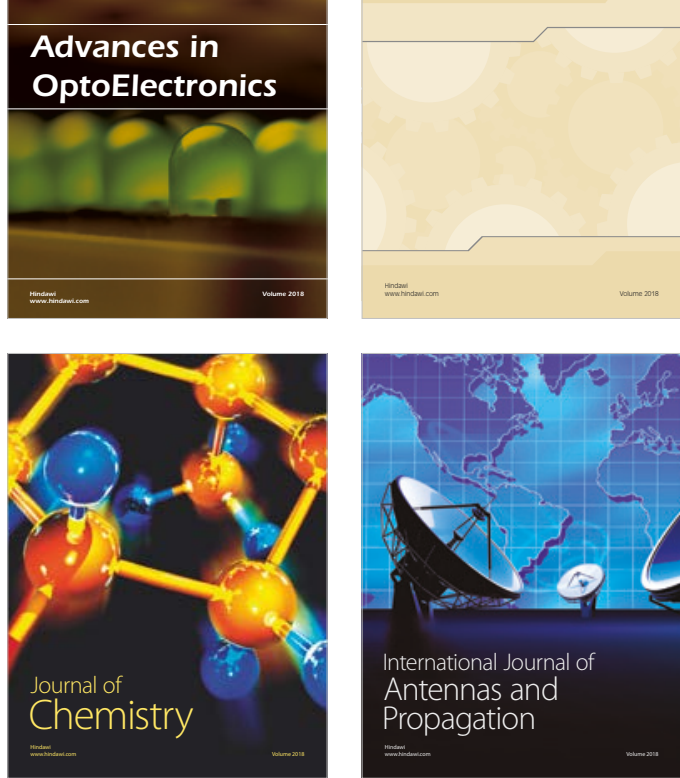

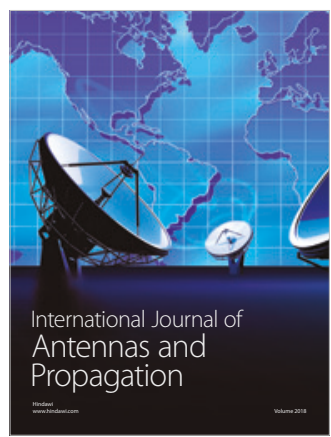

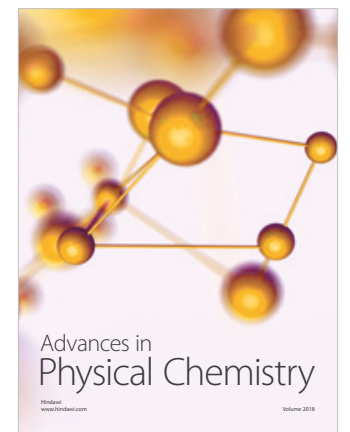

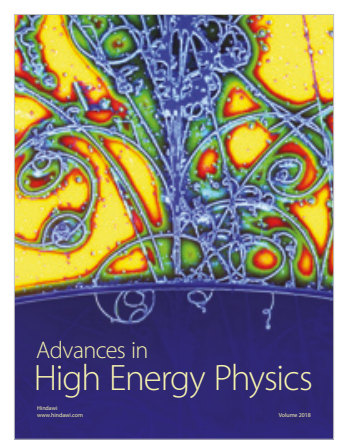

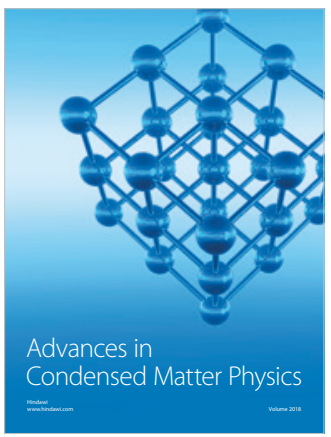

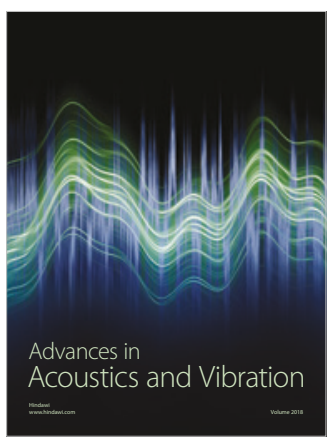

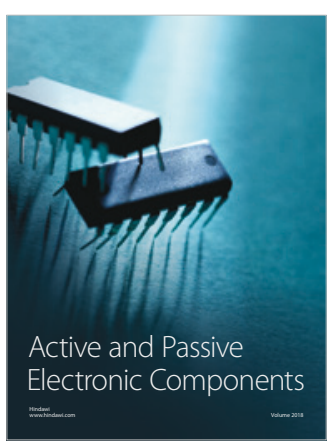
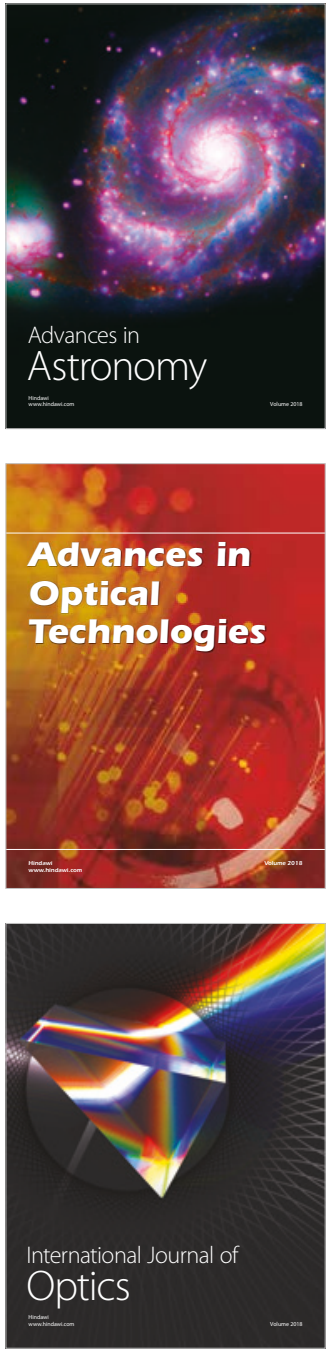